\title{
Transdisziplinäre Realexperimente und künstlerische Forschungspraktiken. Koproduktion urbaner Resilienz im Reallabor Gängeviertel in Hamburg
}

\author{
Michael Ziehl \\ Eingegangen: 31. August 2020 - Angenommen: 12. April 2021 - Online veröffentlicht: 29. April 2021
}

\begin{abstract}
Zusammenfassung
Mittels Reallaboren versuchen Forschende der Raumwissenschaften zunehmend Transformationen urbaner Teilsysteme zu erforschen und aktiv mitzugestalten. Realexperimente nehmen dabei eine zentrale Funktion ein. Als Interventionen in Praxissituationen dienen sie dazu, Möglichkeiten zur Veränderung anzustoßen und zu testen. In diesem Beitrag werden anhand des Reallabors Gängeviertel in Hamburg zwei Realexperimente diskutiert. Diese wurden durchgeführt, um das konfliktbehaftete Kooperationsverfahren zur Sanierung des historischen innerstädtischen Quartiers effizienter zu gestalten und Wissen über divergierende Wertvorstellungen und Handlungsrationalitäten der Beteiligten zu gewinnen. Der Fokus liegt dabei auf künstlerischen Praktiken wie Inszenieren, Arrangieren und Performen, die in das Forschungsdesign integriert wurden. Der Beitrag hat zum Ziel, Potenziale von künstlerischen Praktiken für transformatives Forschen mittels Realexperimenten aufzuzeigen sowie Herausforderungen und Grenzen einer solchen methodischen Erweiterung von Reallaboren zu diskutieren.
\end{abstract}

Schlüsselwörter: Reallabor - Realexperiment künstlerische Forschung - Koproduktion - Urbane Resilienz Hamburg

Dr. Michael Ziehl, CityScienceLab, HafenCity Universität Hamburg, Henning-Voscherau-Platz 1, 20457 Hamburg, Deutschland michael.ziehl@hcu-hamburg.de

(c) (1) (c) 2021 Ziehl; licensee oekom verlag. This Open Access article is published under a Creative Commons Attribution 4.0 International License.

\author{
Transdisciplinary real-world experiments and \\ arts-based research practices. Co-producing \\ urban resilience at Gängeviertel in Hamburg
}

\begin{abstract}
Using real-world laboratories, researchers in spatial sciences increasingly engage in both, shaping and observing transformation processes of urban systems. Thereby, real-world experiments are critical. As interventions into the real, they serve to test and foster possibilities of change. This paper discusses two real-world experiments with reference to the Gängeviertel in Hamburg. Their purpose was to shape the conflictual cooperation process to renovate the historic inner city quarter in a more efficient way and to gain knowledge about motives and values of those who are involved. The paper focusses on arts-based practices such as staging, arranging and performing, that were integrated into the research design with the purpose to outline their potentials for transformative research as well as to discuss challenges and constraints. Furthermore the scientific assumptions of the research design are illustrated.
\end{abstract}

Keywords: Real-world lab - Real-world experiment - Artsbased research - Co-production - Urban resilience - Hamburg

\section{Einleitung}

Realexperimente sind als wissenschaftlich fundierte Interventionen in sozioökologische Prozesse zu verstehen, wobei Forschende mittels gezielter Eingriffe Einfluss auf ihren Untersuchungsgegenstand nehmen. Realexperimente finden oftmals in Praxissituationen statt, an denen unterschiedliche Akteure beteiligt sind. Im Vergleich zu Laborexperimenten gehen sie mit vielen Unsicherheiten einher, da ihr Verlauf, der Erkenntnisgewinn und ihre Wirkungen mehr 
oder weniger unvorhersehbar sind (Schneidewind/SingerBrodowski 2014: 126f.). Für Forschende ist es daher eine Herausforderung, mithilfe von Realexperimenten Transformationen in eine bestimmte Richtung $\mathrm{zu}$ steuern und das dafür notwendige Wissen zu gewinnen. Der vorliegende Beitrag basiert auf der These, dass dabei künstlerische Forschungspraktiken unterstützend wirken können. Sie bieten zwar nicht mehr Sicherheit im Forschungsprozess, sie können aber dazu beitragen, Transformationswissen zu generieren und die Beteiligten so zum Handeln anzuregen, dass diese Transformationen vorantreiben. $\mathrm{Zu}$ dieser These passt es, dass zeitlich parallel zum zunehmenden Interesse der Nachhaltigkeitsforschung an Reallaboren und Realexperimenten auch in der Kunst verstärkt nach Möglichkeiten gesucht wird, gesellschaftliche Entwicklungen mit zu beeinflussen. In vielen Fällen befassen sich Künstlerinnen und Künstler mit möglichen gesellschaftlichen Zukünften und nicht selten sind Städte oder Stadtteile ein zentraler Bezugspunkt ihrer Arbeit (Berger 2018: 13). Dabei agieren sie vielfach als Forschende, die mittels künstlerischer Praktiken neues Wissen erzeugen (Badura/Dubach/Haarmann 2015: 9f). Insofern ist es nicht überraschend, dass zunehmend methodologische Anknüpfungspunkte zwischen künstlerischen Forschungspraktiken und Reallaboren sowie Realexperimenten diskutiert werden (Krohn 2012: $1 \mathrm{ff}$.; Malorny 2018: 82; Ziemer 2019: 102; Matthias/Wildner 2021: o.S.). Schneidewind (2018: 434) geht sogar so weit, Forschen mittels Reallaboren selbst als Teil einer „Zukunftskunst“ zu bezeichnen. Darunter versteht er die Kompetenz der Beteiligten, in transdisziplinären Settings eine nachhaltige Transformation voranzutreiben und das dafür notwendige Wissen zu gewinnen.

In diesem Beitrag geht es um den Einsatz von originär künstlerischen Praktiken wie Inszenieren, Arrangieren und Performen im Rahmen des Reallabors Gängeviertel in Hamburg. Er handelt von den Fragen, welche Herausforderungen für die Forschenden beim Einsatz von künstlerischen Praktiken im Rahmen von Realexperimenten auftreten und wie sie diese bewältigen können. Dazu werden im Anschluss an diese Einleitung die methodischen Zusammenhänge zwischen Reallaboren und Realexperimenten vertieft (Kapitel 2). Danach geht der Beitrag auf die wissenschaftlichen Vorannahmen des Forschungsdesigns ein (Kapitel 3), um dann den Untersuchungsgegenstand darzustellen (Kapitel 4). Darauf aufbauend werden zwei im Reallabor Gängeviertel durchgeführte Realexperimente und die dabei eingesetzten künstlerischen Praktiken beschrieben (Kapitel 5). Darauf folgt eine Darstellung der zentralen Hemmnisse und Herausforderungen des Forschungsdesigns (Kapitel 6) sowie eine Diskussion zum Einsatz von künstlerischen Praktiken im Rahmen von Realexperimenten und Reallaboren (Kapitel 7). Das Fazit fasst zentrale Erkenntnisse zusammen und leistet so einen Beitrag zur Debatte um die methodische Gestaltung von Urbanen Reallaboren in den Raumwissenschaften.

\section{Urbane Reallabore und Realexperimente}

Im Rahmen von Reallaboren geht es neben der Produktion von Wissen über Transformationen in Richtung Nachhaltigkeit um die Gestaltung räumlicher Transformationsprozesse in Zusammenarbeit von wissenschaftlichen und nichtwissenschaftlichen Akteuren (Schneidewind 2018: 447). In Bezug auf Städte erfordert dies, dass Forschende die Beteiligten von urbanen Entwicklungsprozessen so zum Handeln anregen, dass sie Transformationen von Stadträumen vorantreiben und eine nachhaltige urbane Entwicklung unterstützen. Dazu greifen sie auf transdisziplinäre Forschungspraktiken zurück, wobei Transformationsakteure bestimmter städtischer Räume auf verschiedene Weise eingebunden werden. Im Fokus von Reallaboren stehen also aktive Transformationen - verstanden als grundlegende Wandlungsprozesse sozialökologischer Verhältnisse, die auf konkrete Handlungen von bestimmten Akteuren zurückzuführen sind (Schmid 2020: 61). Damit solch ein Wandel mittels Reallaboren vorangetrieben werden kann, müssen die Forschenden ihr Handeln an normativen Grundsätzen ausrichten und versuchen, Transformationen dementsprechend zu steuern. Dies schließt mit ein, dass Forschende die Handlungsrationalitäten und Wertvorstellungen der Beteiligten verstehen lernen. Andernfalls können sie diese kaum zum zielgerichteten Handeln motivieren. Wissen darüber, was den Handlungen von Transformationsakteuren zugrunde liegt und wie ihr Handeln beeinflusst werden kann, wird vor allem mithilfe von Realexperimenten gewonnen, wobei Reallabore einen räumlich und zeitlich eingegrenzten Rahmen vorgeben, um Realexperimente vorzubereiten, durchzuführen und zu evaluieren (Schäpke/Stelzer/Bergmann et al. 2017: 17; Wanner/Hilger/Westerkowski et al. 2018: 102).

Das Generieren von Erkenntnissen orientiert sich dabei an den Wissensformen Systemwissen (1), Ziel- bzw. Orientierungswissen (2) und Transformationswissen (3). Der Prozess der Wissensgewinnung soll darüber hinaus zur Organisation von Lernprozessen unter den Beteiligten dienen. Dieses Lernen gliedert sich idealtypisch in vier Phasen: Problemanalyse (1) auf Basis des Systemwissens, Visionsentwicklung (2) auf Basis des Ziel- bzw. Orientierungswissens, Realexperimente (3) sowie Diffusion und Lernen (4) auf Basis des Transformationswissens (Schneidewind/ Singer-Brodowski 2014: 71; Schäpke/Stelzer/Bergmann et al. 2017: 9). Trotz dieser methodischen Orientierung und zahlreichen aktuellen Forschungen, die auf Reallaboren 
und Realexperimenten fußen, befindet sich die Methode noch in der Entwicklung und Ausdifferenzierung. Offene Fragen bestehen unter anderem in Bezug auf die vielfältigen Rollen von Wissenschaftlerinnen und Wissenschaftlern in Reallaboren sowie ihre Aufgabe, Realexperimente wirksam umzusetzen und Transformationen zielgerichtet zu steuern (Defila/Di Giulio 2018a: $44 \mathrm{ff}$.). Unter anderem ist unklar, auf welchen theoretischen Grundlagen die Ergebnisse von Reallaboren analysiert werden sollten, um Wissen über Transformationsprozesse zu generieren, das zur langfristigen Lösung von Problemen in ebendiesen Prozessen beitragen kann. Bisher werden je nach Disziplin und Untersuchungsgegenstand verschiedene Theorien herangezogen (Schäpke/Stelzer/Bergmann et al. 2017: 46). Im Folgenden werden die wissenschaftlichen Vorannahmen und Theoriegrundlagen des Reallabors Gängeviertel in Hamburg zur Koproduktion urbaner Resilienz dargestellt.

\section{Koproduktion urbaner Resilienz als Teil einer nachhaltigen Transformation}

Urbane Resilienz bezeichnet die Fähigkeit eines urbanen Systems, angesichts von Störungen gewünschte Funktionen aufrechtzuerhalten oder wiederzuerlangen, sich an Veränderungen anzupassen und Systeme schnell zu transformieren, die ihre gegenwärtige und zukünftige Kapazität zur Anpassung einschränken (Meerow/Newell/Stults 2016: 39). Städte sind demnach als komplexe und dynamische urbane Systeme zu fassen, die aus verschiedenen Teilsystemen bestehen. Solche urbanen Teilsysteme sind in besonderem Maße von ihrer gebauten Umwelt in Form von Infrastrukturen und Gebäuden (Wohnungen, Straßen, Bahnschienen, Brücken, Kraftwerken etc.) geprägt (Hassler/Kohler 2014: $119 \mathrm{ff}$.) und können als sozioökologisch-technische Teilsysteme verstanden werden (McPhearson/Pickett/Grimm et al. 2016: 207). Unter Anpassungsfähigkeit lassen sich Maßnahmen zusammenfassen, die die Weiterentwicklung entlang einer eingeschlagenen Richtung ermöglichen. Bei der Transformationsfähigkeit geht es dagegen um neue Entwicklungsrichtungen, die eingeschlagen werden, um grundlegend neue urbane Systeme zu erschaffen (Folke 2016: 9). Das Leitbild der Nachhaltigkeit, wonach ökonomisches Wachstum, soziale Inklusion sowie Umwelt- und Ressourcenschutz im Einklang stehen müssen, ist bei der Umsetzung von Resilienzkonzepten richtungsweisend. Diese Zielrichtung hat in den vergangenen Jahren erheblich an Bedeutung gewonnen, denn ohne diese drohen Anpassungs- und Transformationsprozesse zum Selbstzweck zu werden oder es könnten nichtnachhaltige Folgen in Kauf genommen werden, weil andere Ziele im Vordergrund ste- hen. Zahlreiche Wissenschaftlerinnen und Wissenschaftler bezweifeln allerdings, dass eine nachhaltige Entwicklung angesichts des global dominanten und wachstumsorientierten Wirtschaftssystems derzeit möglich ist (Kopatz 2016: 40; Brand/Wissen 2017: 25; Jackson 2017: 58f.; Engel/ Knieling 2018: 13 ff.; Lessenich 2018: 118). Das gilt auch für Städte, denn aufgrund ihrer ökonomischen Wachstumsabhängigkeit werden zahlreiche Krisen verstärkt, die einer nachhaltigen Entwicklung entgegenstehen - etwa Klimaerwärmung, Umweltverschmutzung, Finanz- und Wirtschaftskrisen sowie soziale Polarisierungen (Eckardt/Brokow-Loga 2020: 15; Schulz/Lange/Hülz et al. 2020: 18). Insofern erscheinen Maßnahmen zu Erhöhung der Resilienz urbaner Teilsysteme nur dann sinnvoll, wenn sie Teil einer nachhaltigen Transformation sind, in deren Zuge auch die Wachstumsfixierung und damit einhergehende Krisen in der Stadtentwicklung überwunden werden.

In den vergangenen Jahren sind in vielen Städten zahlreiche Bürgerorganisationen entstanden, deren Mitglieder durch ihre Tätigkeiten eine nachhaltige Transformation von Städten unterstützen und sie resilienter machen, indem sie die Anpassungs- und Transformationsfähigkeit urbaner Teilsysteme angesichts sozialer, ökonomischer und ökologischer Krisen erhöhen (Frantzeskaki/Haase/Fragkias et al. 2016: IV). Dazu können Wohngruppen, Sozialunternehmen, Kultur- und Bildungseinrichtungen, kleine Genossenschaften oder Urban-Gardening-Vereine zählen. Meines Erachtens erfordert eine resiliente und nachhaltige Stadtentwicklung, dass Politikbetrieb und Verwaltungsapparate die Handlungsfähigkeit und Eigeninitiative von solchen Bürgerorganisationen fördern, damit diese Ressourcen und soziales Kapital besser in die Stadtentwicklung einbringen können (Bovaird/Loeffler 2016: $160 \mathrm{ff}$; Hahne 2018: 50), etwa indem Bürger/-innen Gebäude und Grundstücke nutzen, um Güter und Dienstleistungen selbstorganisiert mit lokalen Ressourcen zu produzieren, und indem Politik sowie Entscheidungsbefugte in der Verwaltung sie durch Genehmigungen, langfristige Besitzrechte und günstige Mieten darin unterstützen. Dies eröffnet Bürgerinnen und Bürgern, Politik und Verwaltung zahlreiche Möglichkeiten, um Anpassungs- und Transformationsprozesse von urbanen Teilsystemen zu gestalten, denn zur Bewältigung aktueller Krisen ist es immer öfter notwendig, dass öffentliche und zivilgesellschaftliche Akteure ihr Handeln aufeinander abstimmen und miteinander kooperieren. Dabei verfolgen die Kooperationspartner/-innen gemeinsame Ziele, die sie alleine nicht erreichen können, weshalb sie ihr Handeln eng koordinieren und zusammenarbeiten (müssen) (Selle 2005: 403; Werner 2012: $70 \mathrm{f}$.). Sofern dabei Güter und Dienstleistungen produziert werden, kann ein solches Zusammenwirken von öffentlichen und zivilgesellschaftlichen Akteuren als Koproduktion ge- 
fasst werden. Der Begriff steht für Prozesse, in deren Verlauf Güter und Leistungen durch Beiträge von Akteuren unterschiedlicher Organisationen hergestellt werden, wobei sie eigene Ressourcen einbringen und Teilbereiche des Koproduktionsprozesses weitgehend unabhängig voneinander kontrollieren (vgl. Ostrom 1996: $1073 \mathrm{ff}$.). In der Stadtentwicklung ist die Bürgerschaft per se Koproduzent, indem sie verschiedene Räume nutzt, Güter und Leistungen produziert und so die Entwicklung von Städten mit beeinflusst (vgl. Selle 2010: 59). Dementsprechend verstehe ich unter der Koproduktion urbaner Resilienz ein Zusammenwirken von Bürgerschaft, Politik und Verwaltung, wodurch Räume und Produkte entstehen, die die Resilienz von urbanen Teilsystemen erhöhen und zu einer nachhaltigen Transformation von Städten beitragen.

Kooperationen zwischen öffentlichen und zivilgesellschaftlichen Akteuren zur gemeinsamen Entwicklung von Stadträumen sind jedoch oftmals konfliktbehaftet. In vielen Fällen hemmen individuelle und strukturelle Aspekte wie Überforderung, abweichende Interessen, unterschiedliche Wertvorstellungen und Handlungsrationalitäten der beteiligten Personen und Organisationen sowie finanzielle und rechtliche Rahmenbedingungen eine produktive und effiziente Zusammenarbeit (Selle 2005: 405; Gualini 2010: 3; Frantzeskaki/Dumitru/Anguelovski et al. 2016: 45; Berger/ Ziemer 2017: 12). Hinsichtlich urbaner Resilienz erfordert die Anpassungsfähigkeit von urbanen Teilsystemen daher auch die Fähigkeit von öffentlichen und zivilgesellschaftlichen Akteuren zur wechselseitigen Anpassung von Handlungen, Verfahrensweisen und Organisationsformen, um produktiver zusammenzuarbeiten. Im Folgenden veranschauliche ich einen solchen Anpassungsprozess anhand der kooperativen Entwicklung des Gängeviertels in Hamburg.

\section{Kooperative Entwicklung des Gängeviertels}

Seit 2010 arbeiten die Stadt Hamburg und die Aktiven des Gängeviertels ${ }^{1}$ zusammen, um das historische Gebäudeensemble zu sanieren und als innerstädtisches Quartier

\footnotetext{
1 Mit „Stadt Hamburg“ bezeichne ich die Gesamtheit der Regierungsorgane, Behörden, öffentlichen Einrichtungen etc. der Freien und Hansestadt Hamburg auf Landesebene und kommunaler Ebene. Unter Aktiven des Gängeviertels verstehe ich Bürger/-innen, die in einem der Gremien des Gängeviertels (Gängeviertel Genossenschaft 2010 eG, Verein Gängeviertel e.V., Arbeitsgruppen) tätig sind oder Räume im Gängeviertel betreiben oder regelmäßig nutzen.
}

mit vielfältigen Nutzungen zu entwickeln. Die Kooperation geht zurück auf die Besetzung der stadteigenen Gebäude im August 2009 durch rund 200 Bürger/-innen, viele von ihnen aus Kunst und Kultur. Mit der friedlichen Besetzung und künstlerischen Aktionen protestierten sie gegen den geplanten Abriss großer Teile der denkmalgeschützten Bausubstanz zugunsten einer gewinnorientierten Projektentwicklung durch einen privaten Investor. Sie forderten stattdessen den kompletten Erhalt und eine gemeinwohlorientierte Nutzung der Gebäude vor allem für kulturelle Zwecke. Nach intensiven Verhandlungen und begleitet von Debatten in Politik und Öffentlichkeit über die Zukunft des Quartiers ließ die Hamburger Stadtentwicklungsbehörde in Zusammenarbeit mit den Aktiven ein Integriertes Entwicklungskonzept erarbeiten. Darin wurden öffentlich geförderte Wohnungen, soziokulturelle Nutzungen, Ateliers und Gewerbeflächen auf rund 7.500 Quadratmetern Nutzfläche vorgesehen (BSU 2010). Die Kosten für die Sanierung wurden auf rund 19,4 Millionen Euro festgelegt, deren Finanzierung vor allem mittels städtebaulicher Förderung des Bundes für Sanierungsgebiete, Haushaltsmittel der Stadt Hamburg sowie Kredite der Hamburgischen Investitions- und Förderbank gesichert wurde. Um eine sachgemäße Verwendung der öffentlichen Gelder und eine effiziente Sanierung sicherzustellen, beauftragte die Stadt Hamburg die Stadterneuerungs- und Stadtentwicklungsgesellschaft Hamburg $\mathrm{mbH}$ (steg) als Sanierungsträgerin mit der Umsetzung. Als Ergänzung schloss die Freie und Hansestadt Hamburg (FHH) mit den neu gegründeten Organisationen der Aktiven (Genossenschaft und gemeinnütziger Verein) 2011 eine Kooperationsvereinbarung zur Umsetzung des Konzepts. ${ }^{2}$ Dennoch kam es im Verlauf der Kooperation zu mehreren Konflikten: Die Aktiven forderten mehr Mitbestimmung während der Sanierung und einen Erbbaurechtsvertrag, der ihnen eine langfristige Selbstverwaltung der Gebäude frühzeitig garantieren sollte. Die Stadt Hamburg ließ sich darauf jedoch zunächst nicht ein, da sie Kostensteigerungen sowie baulichen Mehraufwand fürchtete und die noch jungen Organisationen der Aktiven professionelle Verwaltungsstrukturen erst noch aufbauen mussten. Hinzu kam, dass die Aktiven bereits deutlich mehr Mitsprachemöglichkeiten hatten, als es bei Bauprojekten der öffentlichen Hand in Hamburg üblich ist.

Um das Scheitern der Kooperation zu vermeiden und Zeit für Konfliktlösungen zu gewinnen, vereinbarten die Kooperationspartner/-innen im Jahr 2015 einen Planungsstopp, der rund viereinhalb Jahre dauerte, bis sie sich auf eine Anpassung des Kooperationsverfahrens einigen konnten.

\footnotetext{
2 http://das-gaengeviertel.info/uploads/media/

Kooperationsvereinbarung.pdf (01.03.2021).
} 
Diese Anpassung umfasst eine ganze Reihe von Einzelmaßnahmen, deren zentraler Bestandteil ein Erbbaurechtsvertrag mit 75 Jahren Laufzeit ist. Darin wurde vereinbart, dass die Genossenschaft der Aktiven die von der Sanierungsträgerin fertiggestellten Gebäude als Erbbaurechtsnehmerin eigenständig verwaltet. Dafür zahlt sie einen Erbbauzins von $2 \%$ und verpflichtet sich, das mit der Stadt Hamburg vereinbarte Nutzungskonzept umzusetzen. Im Falle gravierender Verstöße kann die Stadt Hamburg per Heimfallregelung das Erbbaurecht zurücknehmen und eine andere Organisation mit der Verwaltung der Gebäude beauftragen (FHH/GG 2019). ${ }^{3}$

Obwohl urbane Resilienz für das Handeln der Akteure kaum eine Rolle spielt, tragen sie meines Erachtens mittels der kooperativen Sanierung dennoch dazu bei und der Erbbaurechtsvertrag bildet die rechtliche Grundlage, um diese Beiträge zu verstetigen. Indem die Bausubstanz saniert wird und einer kleinteiligen Nutzungsmischung dient, die von den Aktiven langfristig selbstorganisiert und nichtkommerziell umgesetzt wird, tragen sie unter anderem zur funktionalen Diversität, Redundanz und Modularität von mehreren urbanen Teilsystemen in der Hamburger Innenstadt bei und erhöhen so deren Anpassungsfähigkeit (z. B. Kultur-, Verkehrs-, Gebäudesystem) (vgl. Sharifi/Chelleri/Fox-Lent et al. 2017: 17). Gleichzeitig veranschaulicht das Gängeviertel, dass Stadträume in enger Kooperation von Bürgerinnen/Bürgern und Politikerinnen/Politikern entwickelt werden können, und es zeigt eine Alternative zur wachstumsorientierten Stadtentwicklung auf, wie sie vielerorts von Stadtverwaltungen und Planerinnen/Planern vor allem in Zusammenarbeit mit renditeorientierten Immobilienunternehmen umgesetzt wird (Lamker/Schulze Dieckhoff 2020a: 95). Dadurch kann die kooperative Entwicklung des Gängeviertels auch zu einer veränderten Raumvorstellung beitragen, wonach Bürger/-innen selbst aktiver Teil bei der Transformation von Stadträumen sein können, indem sie sich selbst organisieren, engagieren und mit öffentlichen Akteuren kooperieren. So gesehen, kann das Gängeviertel als „Möglichkeitsraum“ (Kagan/Kirchberg/Weisenfeld 2019: 16) zu einer nachhaltigen Transformation beitragen, die über die städtebauliche Transformation des Ortes hinausgeht. Um solch ein outscaling zu unterstützen, ist es aus meiner Sicht wichtig, dass anhand des Kooperationsprozesses übertragbares Wissen darüber gewonnen wird, wie Kooperationsverfahren von öffentlichen und zivilgesellschaftlichen Akteuren gestaltet werden können, damit sie effizient zusammenarbeiten können. Zu diesem Zweck habe ich das Gän-

\footnotetext{
3 http://suche.transparenz.hamburg.de/dataset/ erbbaurechtsvertrag-gaengeviertel-vom-09-08-2019 ?forceWeb=true (01.03.2021).
}

geviertel als Reallabor genutzt und zwei Realexperimente mit Beteiligten der Kooperation durchgeführt, wie ich im Folgenden erläutere.

\section{Das Urbane Reallabor Gängeviertel}

Der Forschungszeitraum, in dem das Gängeviertel als Reallabor genutzt wurde, umfasst etwa 4,5 Jahre und deckt sich weitgehend mit den Verhandlungen zwischen der Stadt Hamburg und den Aktiven des Gängeviertels zur Anpassung des Kooperationsverfahrens. Er beginnt in etwa zeitgleich mit dem Stopp der baulichen Planungen aufgrund von Konflikten in Bezug auf die Sanierung sowie die Selbstverwaltung des Quartiers und endet mit dem Beschluss einer ganzen Reihe von Anpassungsmaßnahmen, deren zentraler Bestandteil der neu abgeschlossene Erbbaurechtsvertrag ist (vgl. Abbildung 1). Insofern wurde ein bereits seit Jahren laufender Kooperationsprozess von mir um ein transformatives Forschungssetting erweitert. Dieses war von Beginn an als Reallabor mit zwei Realexperimenten geplant, wobei das Forschungsdesign mehrfach an die gewonnenen Zwischenergebnisse und den wechselhaften Kooperationsverlauf angepasst wurde. Meine Motivation, das Gängeviertel als Reallabor zu nutzen, entstand aufgrund der sich verschärfenden Konflikte unter den Beteiligten. Aus meiner Sicht drohte die Kooperation zur Sanierung des historischen Gebäudekomplexes zu scheitern, weshalb ich zum einen untersuchen wollte, wie sie sich effektiver gestalten lässt. Zum anderen wollte ich einen Beitrag zur Verbesserung der $\mathrm{Zu}$ sammenarbeit leisten.

Die Untersuchung ist mit dem Gängeviertel als Reallabor auf eine vertiefende Fallstudie eingegrenzt. Da die Kooperation in Intensität und Umfang bisher einmalig ist für die jüngere Stadtentwicklungsgeschichte in Deutschland (Breckner 2015: 196), lässt deren Untersuchung Ergebnisse erwarten, die wegweisend für weitere Kooperationsverfahren sein können. Zur theoriegestützten Analyse der Kooperation wurde ein interdisziplinärer Ansatz gewählt, weil in der Resilienztheorie bisher kein Konzept existiert, mit dem Städte als komplexe und dynamische Systeme ganzheitlich erfasst werden können (McPhearson/Pickett/Grimm et al. 2016: 204). Insbesondere für Machtbeziehungen und -positionen von Akteuren ist die Resilienztheorie weitgehend blind, obwohl diese für die zentralen Aspekte der Anpassung und Transformation von urbanen Teilsystemen in vielen Fällen entscheidend sind (Olsson/Galaz/Boonstra 2014). Daher stützt sich das Forschungsdesign neben Konzepten der Resilienz- und Nachhaltigkeitsforschung auf Raumtheorien des historischgeographischen Materialismus (Belina 2013: 26) und auf Governance-Konzepte, um mit deren Hilfe auch historisch gewachsene Machtstrukturen und Abhängigkeiten zwischen 
08/09 Besetzung des Gängeviertels

12/09 Rückkauf des Gängeviertels

Erste Kooperationsphase

09/10 Integriertes Entwicklungskonzept wird fertiggestellt

11/10 Gründung Gängeviertel Genossenschaft 2010 eG

09/11 Abschluss Kooperationsvereinbarung

Zweite Kooperationsphase

10/11 Gängeviertel wird Sanierungsgebiet

05/12 Übertragung des Gängeviertels an steg, Beginn der Planung

03/13 Sanierungsbeirat wird eingerichtet

10/13 Beginn der Sanierungsarbeiten

08/14 Beginn der Verhandlungen über Selbstverwaltung

01/15 Bezug des ersten sanierten Gebäudes Forschungszeitraum

02/15 Planungsstopp

Dritte Kooperationsphase

10/15 Generalmiet- und Verwaltungsvertrag, Bezug des zweiten sanierten Gebäudes

02/16 Einigung über Betrieb des soziokulturellen Zentrums

03/16 Eröffnung des soziokulturellen Zentrums

04/16 erstes Realexperiment [Bausymposium]

02/17 Verhandlungsabbruch

05/17 zweites Realexperiment [Laborbericht]

11/17 Wiederaufnahme der Verhandlungen

06/19 Abschluss Erbbaurechtsvertrag, Aufhebung Planungsstopp

Wiederaufnahme der Sanierungsplanung

Abbildung 1 Kooperationsprozess und Forschungszeitraum Quelle: Ziehl (2020: 107)

den Beteiligten $\mathrm{zu}$ erfassen. Insgesamt umfasst das Forschungsdesign die vier Aspekte historisch-geographischer Materialismus, Resilienz- und Nachhaltigkeitskonzepte sowie Governance-Theorie als Grundlagen der Analyse (1), den Kooperationsprozess zur Sanierung des Gängeviertels als Gegenstand der Untersuchung (2), die Absicht, die Koproduktion urbaner Resilienz im Zuge der kooperativen Entwicklung des Gängeviertels zu unterstützen als normative Handlungsgrundlage (3) sowie das Reallabor Gängeviertel und zwei transdisziplinäre Realexperimente als Methode der Forschung (4).

Während beim ersten Realexperiment vor allem Wissen über Konflikte im Kooperationsprozess und Lösungsansätze gewonnen wurde, diente das zweite zur Einspeisung von vorläufigen Handlungsempfehlungen und deren Reflexion durch die Beteiligten in Bezug auf ihr Wirkungspotenzial und ihre Realisierungschancen. Dieses Feedback wurde im weiteren Forschungsprozess herangezogen, um abschließende Handlungsanregungen zu generieren, die über die Kooperation zur Sanierung des Gängeviertels hinaus bei der Koproduktion urbaner Resilienz anwendbar sind (Ziehl 2020: 170). Damit verfolgte ich das Ziel, Transformationswissen zu gewinnen und zu veröffentlichen, das einen ,weitergehenden Beitrag zur gesellschaftlichen Transformation leisten“ (Schäpke/Stelzer/Bergmann et al. 2017: 25) kann. Bei beiden Realexperimenten habe ich künstlerische Praktiken eingesetzt, wie ich im folgenden Kapitel ausführe. Damit beabsichtigte ich, bestehende Konflikte und Potenziale der Kooperation nicht nur in Begriffen, Worten und
Konzepten zu beschreiben und zu vermitteln, wie es in der Wissenschaft üblich ist, sondern für die Beteiligten auch ästhetisch erfahrbar zu machen (Hildebrandt 2014: 75). Damit waren zwei Ziele verbunden: Erstens sollten die von mir eingesetzten künstlerischen Praktiken zur ganzheitlichen Betrachtung des Kooperationsprozesses beitragen, indem auch die subjektive Ebene der Beteiligten adressiert und untersucht wird. So wollte ich Wissen über ihre persönlichen Handlungsrationalitäten und Wertvorstellungen in Bezug auf den Kooperationsprozess gewinnen (Heinrichs 2018: 132). Zweitens beabsichtigte ich, mittels der von mir kreierten Situationen Handlungsblockaden bei den Beteiligten zu lösen und sie zu kooperativem Handeln anzuregen, indem sie ihre jeweiligen Handlungsrationalitäten und Wertvorstellungen untereinander besser verstehen lernen und Empathie aufbauen.

\subsection{Erstes Realexperiment: Bausymposium}

Als erstes Realexperiment habe ich ein Symposium zum Kooperationsverfahren und zur Sanierung im Gängeviertel umgesetzt. Dessen Hauptteil bestand in der ,Werkstatt für gemeinsame Zukünfte“, bei der relevante Akteure des Verhandlungsgeschehens seitens der Aktiven und der Stadt Hamburg den bisherigen Kooperationsverlauf sowie mögliche zukünftige Entwicklungen unter Anleitung eines professionellen Moderators gemeinsam reflektierten (vgl. Abbildung 2). Der Workshop war eine sorgfältige „Inszenierung" (Seel 2011: 352) im Sinne eines absichtsvoll herbeigeführten Prozesses unter Einbindung eines Publikums. Ich verfolgte damit die Absicht, dass die Anwesenden in der Rolle eines teilnehmenden Publikums einen unmittelbaren Eindruck von der Ästhetik des Ortes und seiner Geschichte bekommen. Daher habe ich den Workshop im bereits sanierten Seminarraum des Gängeviertels veranstaltet, denn dort lässt sich die Geschichte des Gebäudes von seiner Entstehung bis zur Sanierung anhand der verbauten Materialien deutlich erkennen. Außerdem habe ich verschiedene Gegenstände, Möbel und Bilder mit Bezug zum Gängeviertel sorgfältig im Raum ,arrangiert“ (Siegmund 2015: 139), wobei sowohl die von mir gewählten Gegenstände wie auch die eingeladenen Akteure als Bestandteile des Arrangements zu verstehen sind (Siegmund 2015: 139). Insgesamt sollte den Teilnehmenden so der Gegenstand ihrer Zusammenarbeit - das Gängeviertel - vergegenwärtigt werden. Darüber hinaus wurden die Objekte von mir so ausgewählt, dass sie über den Ortsbezug hinaus auf weitere Kontexte (z. B. Kunstproduktion und Stadtentwicklung) verwiesen, da diese bei den Debatten um die Sanierung des Gängeviertels eine zentrale Rolle spielen. Gleichzeitig wollte ich eine gemütliche „Atmosphäre“ (Böhme 2011: 243) schaffen - verstanden als individuelle und emotionale Wahrnehmung ei- 


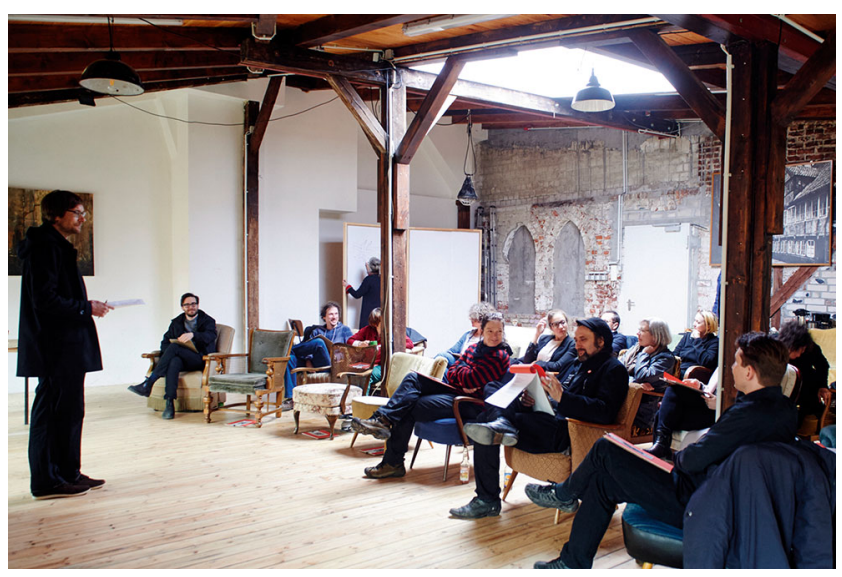

Abbildung 2 Werkstatt für gemeinsame Zukünfte Foto: Franziska Holz

nes räumlichen Settings durch die Teilnehmenden (Böhme 2011: 243). Diese sollte sich absichtlich von den eher kalten und streng geordneten Konferenzräumen in den Behörden unterscheiden, wo die Kooperationsakteure üblicherweise die Zukunft des Gängeviertels verhandelten. Eine aufgelockerte Sitzanordnung aus unterschiedlichen Sesseln sollte außerdem dazu beitragen, dass sich die Teilnehmenden als individuelle Personen und nicht primär als Repräsentantinnen/Repräsentanten einer Institution adressiert fühlen. So sollte die Empathie der Teilnehmenden und ihr Bezug zum Gängeviertel gefördert werden, um unterschiedliche Wertvorstellungen, die sie daran knüpfen, während des Workshops produktiv verhandeln zu können. Darauf aufbauend sollten gemeinsame Ziele für die Zukunft des Gängeviertels gefunden und Handlungsblockaden zumindest teilweise gelöst werden, die eine Kompromissfindung im Konflikt um die Selbstverwaltung behinderten. Insgesamt beabsichtigte ich also mithilfe der künstlerischen Praktiken des Inszenierens und Arrangierens ,ästhetische Erfahrungen“ (Bippus 2009: 12) unter den teilnehmenden Akteuren zu fördern, die eine zwischenmenschliche Annäherung und eine produktive Bezugnahme zum Gegenstand ihrer Kooperation unterstützen.

Das beim Workshop gewonnene Ziel- und Orientierungswissen zeigt, dass zum damaligen Zeitpunkt eine Anpassung des Kooperationsverfahrens notwendig war, um es für die Beteiligten erfolgreich zu gestalten. Dazu zählt unter anderem, dass die Selbstverwaltung des Gängeviertels geklärt und das Integrierte Entwicklungskonzept in Bezug auf die Nutzung der zu sanierenden Flächen überarbeitet wird. Außerdem benannten Vertreter/-innen beider Seiten soziale Defizite bei der Zusammenarbeit. Demnach mangelte es beiderseits an Vertrauen, Wertschätzung und Kompromissbereitschaft. Dies deutet auf erhebliche soziale Potenziale zur Anpassung der Kooperation an Konflikte hin. Um sie zu nutzen, müssten die Kooperationspartner allerdings zunächst ihre Zielvorstellungen und Wertmaßstäbe in Bezug auf die Sanierung des Gängeviertels untereinander abstimmen, wie der Workshop ebenfalls gezeigt hat.

Mehrere Teilnehmer/-innen bezeichneten die Wirkung des Workshops im Anschluss als ,therapeutisch“. Insofern kann davon ausgegangen werden, dass die zuvor beschriebenen künstlerischen Praktiken durchaus zu einer zwischenmenschlichen Annäherung beitragen konnten und sich die Teilnehmer/-innen wie beabsichtigt ein Stück weit aus ihren repräsentativen Rollen als Vertretungen zweier Verhandlungsparteien gelöst haben. Gestützt wird dieser Rückschluss dadurch, dass die Stadt Hamburg und das Gängeviertel infolge des Bausymposiums die Art und Weise veränderten, wie sie miteinander verhandelten. Unter Anleitung einer professionellen Moderatorin fanden an einem neutralen Ort moderierte Arbeitstreffen statt. So sollte die interne Kommunikation verbessert und das Vertrauen zwischen den Verhandelnden gestärkt werden. Das deutet auf einen Lernprozess bei den Kooperationspartnerinnen/partnern über den Umgang miteinander hin. Allerdings führten die Maßnahmen nicht zu einer Einigung. Diese gelang erst nach einem Neustart der Verhandlungsgespräche, der weitgehend unabhängig von meinem Realexperiment erfolgte.

\subsection{Zweites Realexperiment: Laborbericht}

Als zweites Realexperiment habe ich im Mai 2017 eine Broschüre mit dem Titel ,Zukunftsfähigkeit durch Kooperation - ein Laborbericht aus dem Gängeviertel" veröffentlicht und präsentiert (Ziehl 2017). Ziel war es, damit ein boundary object zu kreieren. Solche Grenzgegenstände dienen dazu, zwischen unterschiedlichen Akteuren zu vermitteln und zum Austausch ihrer Wertvorstellungen und Handlungsrationalitäten anzuregen, indem sie deren Wissensbestände zusammenführen und ,aufgrund ihrer Plastizität eine Verständigung über kognitive und normative Grenzen hinweg ermöglichen“ (Schäfer 2013: 181). In Frage kommen dafür materielle und immaterielle Entitäten wie Alltagsvorstellungen, Ideen, Konzepte, Artefakte und Publikationen (Schäfer 2013: 182). Schneidewind und Scheck (2013: 240) schlagen vor, im Rahmen von transformativen Forschungen einzelne Städte als boundary objects zu nutzen, indem sie als räumliche Bezugsrahmen herangezogen werden. Dies erschien mir im Rahmen meiner Forschung allerdings nicht praktikabel, da divergierende Vorstellungen der Beteiligten in Bezug auf eine wünschenswerte Stadtentwicklung in Hamburg für öffentlich ausgetragene Konflikte sorgte und ihre Zusammenarbeit zusätzlich erschwerte. Während die Stadt Hamburg einer stark auf wirtschaftliches Wachstum ausgerichteten Stadtentwicklungspolitik als Leitbild folgte, forderten die 
Aktiven des Gängeviertels unter dem Slogan „Recht auf Stadt" eine Politik, die weniger auf ökonomische Interessen von Investoren ausgerichtet ist und sich stärker an den Alltagsbedürfnissen von Stadtbewohnerinnen/-bewohnern orientiert (Novy/Colomb 2013: 1827).

Um solche Konflikte zu überbrücken, eignen sich laut Meerow, Newell und Stults (2016: 46) insbesondere Konzepte urbaner Resilienz als boundary objects, da diese Städte als grundsätzlich anpassungsfähige Systeme gefasst werden können, die von verschiedenen Akteuren mitgestaltet werden. Demnach sei urbane Resilienz als Konzept geeignet, um zwischen divergierenden Interessen in Bezug auf die Stadtentwicklung zu vermitteln. Dies hätte im Fall des Gängeviertels allerdings erfordert, dass urbane Resilienz mit den Beteiligten als erstrebenswertes Ziel zunächst definiert wird, denn das Konzept hatte kaum Bedeutung für ihr Handeln. Da es den Beteiligten zu dem Zeitpunkt weitgehend unbekannt war, ging es ihnen nicht primär darum, Hamburg resilienter zu machen. Mit der Broschüre habe ich mich daher für eine materielle Form als boundary object entschieden, die auf eine gelingende Kooperation fokussiert und dafür die Ergebnisse der ,Werkstatt für gemeinsame Zukünfte“, meine wissenschaftliche Interpretation des Kooperationsprozesses und daraus abgeleitete Handlungsempfehlungen zur Diskussion stellt. Die Broschüre sollte über die von mir geschaffene Workshop-Situation hinaus als Bezugsobjekt für die Kooperationsakteure dienen, zu Feedback anregen sowie auf gemeinsame Erfolge, Konflikte und Lösungsvorschläge verweisen. Indem Exemplare davon unter anderem an die Beteiligten verteilt wurden, sollten die Verhandlungen zur Anpassung des Kooperationsverfahrens unterstützt werden. Diese Akte des unabhängigen Selbstpublizierens meiner Forschung waren als Beiträge zur fachlichen Debatte über die Kooperation von öffentlichen und zivilgesellschaftlichen Akteuren und zur Einspeisung von Zwischenergebnissen meiner Forschung in den Kooperationsprozess gedacht. Dies kann darüber hinaus als eine eigenständige künstlerische Praktik im Rahmen des zweiten Realexperiments verstanden werden, weil es mir nicht bloß darum ging, Erkenntnisse zu veröffentlichen, sondern auch einen Gegenstand zu verbreiten, der auch mittels seiner Ästhetik und den versammelten Inhalten Vermittlungsprozesse zwischen den Kooperationsakteuren unterstützt (Gilbert 2016: 7).

Die Präsentation der Broschüre und der anschließende Workshop zu den darin enthaltenen Handlungsempfehlungen fanden in einem Seminarraum der HafenCity Universität Hamburg (HCU) statt. Eingeladen waren insbesondere Beteiligte der bisherigen Verhandlungen zwischen dem Gängeviertel und der Stadt Hamburg. Die Wahl einer Universität als Veranstaltungsort erfolgte in der Absicht, die Funktion der Veranstaltung als Experiment zur Wissensge- winnung zu unterstreichen. Um dies mithilfe von künstlerischen Praktiken auch ästhetisch erlebbar zu machen, wurde der Seminarraum durch die Wahl von abwaschbaren Materialien und einer strengen, reduzierten Anordnung von Mobiliar in Anlehnung an naturwissenschaftliche Laborräume inszeniert. An den Wänden des Seminarraums hingen die von mir erarbeiteten vorläufigen Handlungsempfehlungen zur Verbesserung der Kooperation und die Teilnehmenden waren aufgefordert, deren Praxistauglichkeit einzuschätzen, indem sie schriftliche Anmerkungen auf Haftzetteln ergänzten. Als Teil der Inszenierung trat ich in einer Kleidung auf, die Laborschutzanzügen nachempfunden war und trug den wissenschaftlichen Hintergrund meiner Forschung vor. Mithilfe dieses „Vortrags als Performance“ (Peters 2011: 14) - verstanden als transdisziplinäres „Ineinandergreifen von Forschung und Darstellung" (Peters 2011: 14) - stellte ich auch meine Doppelrolle im Reallabor als Handelnder und Forschender offensiv dar. Gleichzeitig adressierte ich die Teilnehmenden als relevante Koproduzierende von Wissen im Rahmen des zweiten Realexperiments. So wollte ich sie zu einem offenen Feedback in Bezug auf die vorläufigen Handlungsempfehlungen anregen, das vor allem auf ihren persönlichen Ansichten beruhte und nicht primär auf den Interessen der von ihnen repräsentierten Organisationen. Im Fokus des zweiten Realexperiments standen insofern die von mir aus meiner Doppelrolle heraus gewonnenen vorläufigen Handlungsempfehlungen zur Verbesserung des Kooperationsverfahrens sowie das Feedback der Teilnehmenden dazu.

Das auf diese Weise gewonnene Transformationswissen zeigt unter anderem, dass laut den Teilnahmeergebnissen höhergestellte Entscheider/-innen aus Politik und Verwaltung direkt in das Kooperationsverfahren eingebunden werden sollten. Begründet wurde dies damit, dass die Vertreter/-innen der Stadt Hamburg in Verhandlungstreffen keine ausreichende Entscheidungskompetenz hätten, um einen Kompromiss mit den Aktiven des Gängeviertels zu vereinbaren. Dies konnte ich mithilfe der von mir publizierten Broschüre als ein Zwischenergebnis meiner Forschung in den weiteren Verhandlungsprozess einspeisen und so zur Einführung eines Koordinierungskreises anregen, der die operative Ebene zur Planung und Ausführung von Sanierungsmaßnahmen mit der Entscheidungsebene in den beteiligten Organisationen enger verknüpft. Dass es dabei nicht bloß um formale Entscheidungsprozesse geht, hatte bereits das erste Realexperiment ergeben. Demnach ist es notwendig, dass Entscheider/-innen beider Seiten persönlich zusammenkommen und Verständnis für die jeweiligen Handlungsrationalitäten und Wertvorstellungen als Basis einer vertrauensvollen und produktiven Zusammenarbeit entwickeln. Der Koordinierungskreis bildet dafür einen organisatorischen Rahmen, der das wechselseitige Lernen unter den Beteiligten 
dauerhaft fördert und somit das Finden von gemeinsamen Lösungen für Probleme im Kooperationsprozess unterstützt. Ich sehe darin auch einen Beitrag zur Koproduktion urbaner Resilienz, denn das Gremium kann das Aushandeln weiterer Anpassungen im Kooperationsverfahren unterstützen, falls dies aufgrund von Konflikten notwendig werden sollte, und somit zu einer langfristig effizienten Zusammenarbeit beitragen.

\subsection{Zentrale Hemmnisse und Herausforderungen des Forschungsdesigns}

Die Realexperimente wurden von mir alleine konzeptioniert und basierten auf meinen Vorannahmen bezüglich der Konflikte im Kooperationsprozess sowie dem von mir identifizierten Potenzial zur Koproduktion urbaner Resilienz. Für die Durchführung war ich allerdings auf die Zusammenarbeit mit Kooperationsakteuren angewiesen, die meine Perspektive nicht teilten. Diese Konstellation führte zu erheblichen Hemmnissen im Forschungsprozess, die ich im Folgenden darstelle und reflektiere.

\subsubsection{Doppelrolle als Aktiver und Forschender}

Auf meine Einladung zum ersten Realexperiment reagierten die Vertreter/-innen der Stadt Hamburg skeptisch und lehnten ihre Teilnahme zunächst ab. Sie befürchteten, dass vertrauliche Inhalte aus den Verhandlungen zwischen der Stadt Hamburg und dem Gängeviertel öffentlich und sie als Blockierende dargestellt werden könnten (Sanierungsbeirat Gängeviertel 2016: 3). Um sie dennoch zur Teilnahme zu bewegen, habe ich das erste Realexperiment modifiziert, indem der Großteil unter Ausschluss der Öffentlichkeit durchgeführt und Workshop-Ergebnisse nur in Abstimmung veröffentlicht wurden. Das skeptische Verhalten seitens der Stadt hängt maßgeblich mit der Doppelrolle zusammen, die ich im Forschungsprozess innehatte, denn auch als Teil der Besetzerinitiative, Vorsitzender im Aufsichtsrat der Gängeviertel Genossenschaft 2010 eG und Mitglied in der Verhandlungs-AG habe ich den Kooperationsprozess mitgestaltet. Insofern bin ich sowohl als Aktiver des Gängeviertels wie auch als Forschender aufgetreten. ${ }^{4}$ In beiden Rollen habe ich mich dafür eingesetzt, dass es den Akteu-

\footnotetext{
${ }^{4}$ In vielen Fällen nehmen Forschende bei Realexperimenten mehrere Rollen ein. Diese können das Initiieren, Moderieren, Analysieren und Reflektieren von Prozessen sowie die Beratung der Beteiligten umfassen (Beecroft/Trenks/Rhodius et al. 2018: 90). Solche Mehrfachrollen werden einem turn to action zugeschrieben, in dessen Zuge Forschende und Planer/-innen zunehmend versuchen, urbane Transformationsprozesse aktiv zu unterstützen (Lamker/ Schulze Dieckhoff 2020b: 381).
}

ren gelingt, die Selbstverwaltung des Gängeviertels mit der langfristigen Entwicklung in Einklang zu bringen und das Kooperationsverfahren dementsprechend anzupassen. Diese Beweggründe für mein Handeln habe ich den Beteiligten wiederholt dargelegt, um ihnen gegenüber transparent und fair in Bezug auf meine Ziele und ihre Funktion als Koproduzierende von Wissen beim Erreichen dieser Ziele zu sein (Schneidewind/Singer-Brodowski 2014: 72). Dennoch hat meine Einbindung in die Selbstverwaltungsstrukturen die Sorge ausgelöst, dass ich verzerrtes Wissen publizieren könnte, indem ich aktivistisch motivierte Bewertungen vornehme. In der Folge habe ich mich umso mehr um eine ausgewogene und wissenschaftlich fundierte Analyse des Kooperationsprozesses bemüht - zum einen, um den Ansprüchen wissenschaftlicher Forschung gerecht zu werden, zum anderen, damit meine Anregungen zur Anpassung des Kooperationsverfahrens sowohl seitens des Gängeviertels wie auch der Stadt Hamburg ernst genommen werden. Zur Einspeisung von Handlungsempfehlungen hat sich meine Doppelrolle sogar als entscheidender Vorteil erwiesen, weil ich als Teil der Verhandlungsgruppe Zwischenergebnisse meiner Forschung auch in Verhandlungsgespräche einbringen konnte.

\subsubsection{Anpassung und Ko-Design im Forschungsprozess}

Zum Zeitpunkt des zweiten Realexperiments hatte die Stadt Hamburg alle Verhandlungen und Gespräche mit den Aktiven des Gängeviertels ausgesetzt. Begründet wurde dies damit, dass interne Beratungen und ressortübergreifende $\mathrm{Ab}$ stimmungen zwischen Verwaltung und Politik notwendig seien, bevor weiterverhandelt werden könne. Hinzu kam, dass der bevorstehende G20-Gipfel im Juli 2017 in Hamburg die Verhandlungen überschattete. Die Stadt wollte in dessen Vorfeld keine weiteren Gespräche mit Vertreterinnen/Vertretern des Gängeviertels führen, um sich nicht öffentlich angreifbar zu machen, weil befürchtet wurde, dass es im Zuge der erwarteten Gegenproteste zu gewalttätigen Aktionen auch aus dem Gängeviertel kommen könnte (Bruns 2018: 59). Daher nahmen am zweiten Realexperiment keine Vertreter/-innen der Stadt Hamburg teil, sondern vor allem Aktive des Gängeviertels und Angehörige der HafenCity Universität Hamburg. Das dabei gewonnene Wissen ist dementsprechend lückenhaft. Daran wird deutlich, dass es notwendig sein kann, Realexperimente an den Handlungszwängen der Beteiligten auszurichten, um ihre Teilnahme abzusichern. Dies kann gelingen, indem sie frühzeitig eingebunden werden und das Forschungsdesign sowie die Realexperimente gemeinsam entwickelt werden. Durch solch ein Co-Design können die Forschenden dafür sorgen, dass die Beteiligten verbindlicher mitwirken, die Forschungsergebnisse eher akzeptieren und weitergehend anwenden (Page/Wise/Lindenfeld et al. 2016: 86). Damit 
einher geht meines Erachtens allerdings auch das Risiko, dass eine Forschung weniger transformativ ausfällt, wenn Beteiligte am Status quo festhalten möchten. Daher ist es von zentraler Bedeutung, dass die Ergebnisse von Reallaboren wissenschaftlich nachvollziehbar aufgearbeitet und unabhängig von den Interessen der Beteiligten durch die Forschenden publiziert werden können. So kann das koproduzierte Wissen epistemologisch abgesichert und öffentlich zugänglich gemacht werden. Dies war beim Reallabor Gängeviertel sichergestellt, da die Realexperimente zentrale Bestandteile meiner Promotion waren (Ziehl 2020).

\subsection{3 Überraschungen und Nichtwissen}

Bei der Durchführung von Realexperimenten zählt der Umgang mit Nichtwissen und möglichen Überraschungen zu den zentralen Herausforderungen, da Forschende die Bedingungen von Realexperimenten niemals vollständig kontrollieren können (Schneidewind/Singer-Brodowski 2014: 126). Im Reallabor Gängeviertel überraschte vor allem die Verweigerungshaltung der Stadt Hamburg. Daraus konnten Erkenntnisse über das Verwaltungshandeln generiert werden in Bezug auf das als zu aktivistisch empfundene Forschungsdesign und den Umgang mit Öffentlichkeiten. In solchen Überraschungen liegt aus soziologischer Sicht der eigentlich experimentelle Charakter von Realexperimenten, weshalb sie die Kompetenz der Beteiligten erfordern, aus unkontrollierbaren Situationen zu lernen und produktiv mit Nichtwissen und Überraschungen umzugehen, um Transformationen in eine gewünschte Richtung zu steuern (Groß 2017: 22). ${ }^{5}$ Um dies leisten zu können, sollten Realexperimente so in Reallabore eingebunden werden, dass neben experimentellen Interventionen auch eine planbare Beeinflussung möglich ist. Im Fall des Reallabors Gängeviertel war dies durch meine direkte Einbindung in das Verhandlungsgeschehen möglich, wie ich bereits beschrieben habe.

\footnotetext{
${ }^{5}$ Genau darum geht es aus meiner Sicht auch bei der Herstellung urbaner Resilienz. So beziehen vor allem Überlegungen zur generellen Anpassungsfähigkeit von Städten den produktiven Umgang mit Nichtwissen ausdrücklich mit ein (Meerow/Newell/ Stults 2016: 46). Der Umweltsoziologe Matthias Groß argumentiert zwar, dass sich Realexperimente und Resilienz-Konzepte weitgehend ausschließen, weil das Resilienz-Konzept lediglich auf den Erhalt eines Status quo ausgerichtet sei, wohingegen Realexperimente der Überwindung bestehender Strukturen und Funktionen von urbanen Teilsystemen dienen sollen (Groß 2014: 165). Dieser Gegensatz gilt meines Erachtens aber nicht, wenn ResilienzKonzepte mit einer nachhaltigen Transformation zusammen gedacht werden wie im Fall der hier vorgestellten Forschung und wie sie sich in den vergangenen Jahren in raumbezogenen Diskursen durchgesetzt haben.
}

\subsubsection{Zeitrahmen für Reflexion und Transformation}

Wie meine Erfahrung zeigt, sollten Forschende die für Reallabore charakteristischen Bereiche Wissensgenerierung, Transformation und Lernprozess nach Möglichkeit so entkoppeln, dass ein Scheitern in einem Bereich nicht das Scheitern in den anderen Bereichen nach sich zieht. Zwar können Realexperimente im wissenschaftlichen Sinne kaum Scheitern, denn sobald überraschendes Wissen erzeugt wird, sind sie erfolgreich verlaufen, auch - oder gerade - wenn die Vorannahmen der Forschenden nicht bestätigt werden. Reallabore, mit denen Transformationsund Anpassungsprozesse erwirkt werden sollen, können jedoch in einem gesellschaftlichen Sinne scheitern, wenn sie nicht zu einer beabsichtigten Veränderung beitragen (Groß 2014: 8; Groß 2017: 22). Dies wurde insbesondere beim zweiten Realexperiment deutlich: Weil die Vertreter/-innen der Stadt daran nicht teilnahmen, konnte das Experiment bei ihnen keine direkte Wirkung entfalten. ${ }^{6}$ Um dem entgegenzuwirken, sollten Realexperimente als punktuelle Interventionen langfristig in Reallabore eingebunden werden, die ausreichend Zeit und Ressourcen bieten, damit Forschungsdesigns an den Belangen und Handlungszwängen der Beteiligten ausgerichtet und gegebenenfalls angepasst werden können. Daraus ergibt sich auch die Möglichkeit, neben der Wirkung von Realexperimenten andere Einflussfaktoren auf Transformationsprozesse wie etwa veränderte Rahmenbedingungen über einen längeren Zeitraum zu erfassen.

Darüber hinaus zählt zu einer ,guten Gestaltung“ (Krohn 2012: 12) von Realexperimenten, dass die Forschenden ihre Interventionen in Bezug auf die damit verfolgten Ziele und den tatsächlichen Prozessverlauf methodisch reflektieren. Dafür sollten Reallabore so konzipiert werden, dass die genauen Wirkungszusammenhänge von Realexperimenten über einen längeren Zeitraum erfasst werden können (Defila/Di Guilio 2018b: 24). Im Fall des Reallabors Gängeviertel war diese Analyse im Rahmen der zur Verfügung stehenden Zeit und Ressourcen nur bedingt möglich. Um den kausalen Zusammenhang zwischen den Realexperimenten und ihrem Beitrag zur Anpassung des Kooperationsverfahrens zu verifizieren, wäre weitere Forschung notwendig gewesen, etwa in Form von nachträglichen Interviews mit den Teilnehmenden. Trotz dieser Wissenslücken schließe ich aus der Prozessbeobachtung und dem Feedback der Beteiligten,

\footnotetext{
${ }^{6}$ Zwar wäre es aussichtsreich gewesen, wenn das zweite Realexperiment später stattgefunden hätte. Allerdings waren aufgrund der Einbettung meiner Promotion in das Graduiertenkolleg „Performing Citizenship“ die Zeitpunkte meiner beiden Realexperimente als künstlerisch-praktische Forschungsteile von der Kollegsleitung vorgegeben.
} 
dass Forschende Transformations- und Anpassungsprozesse von urbanen Teilsystemen wirksam mitgestalten können, indem sie wie in meinem Fall künstlerische Praktiken in Realexperimente einbinden. Dies erörtere ich im Folgenden anhand meiner Forschungserfahrung und ordne mein Forschungsdesign methodologisch ein.

\section{Künstlerische Praktiken im Rahmen von Realexperimenten und Reallaboren}

Mittels künstlerischer Praktiken im Rahmen von Realexperimenten und Reallaboren kann Realität nicht nur wie in der Wissenschaft üblich in Begriffen, Worten und Konzepten beschrieben und vermittelt werden, sondern für die Beteiligten auch erfahrbar gemacht werden (Hildebrandt 2014: 75). So können künstlerische Praktiken wie beim Reallabor Gängeviertel zu einer ganzheitlichen Betrachtung von Transformations- und Anpassungsprozessen beitragen. Dabei steht aufgrund der individuellen Wahrnehmung von Erfahrungen insbesondere die subjektive Ebene der Beteiligten im Fokus (Heinrichs 2018: 132). Mittels geeigneter Forschungssettings lassen sich so Erkenntnisse unter anderem über die Handlungsrationalitäten und Wertvorstellungen der Beteiligten gewinnen. In vielen Fällen kann dabei schon während der Vorbereitung von künstlerischen Eingriffen Wissen gewonnen werden, denn dies erfordert oft forschendes Handeln und die Kommunikation mit Beteiligten. Die wesentlichen Potenziale von künstlerischen Praktiken für transformatives Forschen mit Reallaboren und Realexperimenten begründen sich allerdings darin, dass Forschende die Wahrnehmung der Beteiligten gezielt beeinflussen können, indem sie ästhetische Erfahrungen inszenieren und soziale Situationen kreieren. In solchen Situationen können relevante Akteure von Transformationsprozessen von den Forschenden gezielt in Bezug zu konkreten Problemen und Lösungsansätzen gesetzt und diese Relationen für die Beteiligten selbst erfahrbar gemacht werden. Auch ist eine (Re)kontextualisierung mit Debatten und Diskursen möglich, die in Bezug auf die jeweiligen Transformationsprozesse relevant sind, etwa indem diese mittels (Lecture-)Performances kommuniziert werden. Im Idealfall können Akteure auf diese Weise so zum Handeln angeregt werden, dass sie Transformationen in Richtung einer nachhaltigen Entwicklung aktiv unterstützen. In Bezug auf die Koproduktion urbaner Resilienz kann das beispielsweise bedeuten, dass über künstlerische Praktiken zwischen zivilgesellschaftlichen und öffentlichen Akteuren Empathie, Vertrauen und Lernbereitschaft gefördert werden. Individuelle ästhetische Erfahrungen können dies unterstützen, indem sie zum Hinterfragen von Wertvorstellungen und Grundannahmen bei den Beteiligten anregen. Darauf aufbauend können sie das Lösen von Handlungsblockaden unterstützen und die Kooperationsbereitschaft erhöhen.

Da Realexperimente auf wissenschaftlich gestützten Vorannahmen basieren, anhand derer Ergebnisse interpretiert und Wirkungen beurteilt werden, sind sie von rein künstlerischen Interventionen zu unterscheiden, denn deren Ausgang ist in der Regel offen angelegt und sie beruhen nicht primär auf wissenschaftlichen Thesen (Frahm 2015: 166). Abzugrenzen sind Realexperimente ebenso vom reinen Ausprobieren im Sinne von try and error. Daran wird deutlich, dass sich die Forschenden beim Einsatz von künstlerischen Praktiken im Rahmen von Realexperimenten nicht nach den sehr offenen Interpretationsspielräumen einer freien Kunstproduktion richten können (Holub 2015: 35). Im Gegenteil geht es beim Forschen mittels Reallaboren und Realexperimenten um praxisbezogene Beiträge zur Lösung von gesellschaftlichen Problemen anhand klar definierter Zielsetzungen. Gleichzeitig sind Realexperimente aufgrund der dafür notwendigen normativen Handlungsgrundlagen der Forschenden nicht mit dem Wissenschaftsideal einer vermeintlich objektiven Forschung vereinbar. ${ }^{7}$ Stattdessen oszillieren solche Realexperimente zwischen künstlerischer und wissenschaftlicher Forschung und werden mithilfe von Praktiken aus beiden Bereichen als Teile einer pluralen Methodik umgesetzt (Haarmann 2019: 287), um lösungsorientiertes Wissen zu generieren und Transformationen sowie Anpassungen urbaner Teilsysteme aktiv mitzugestalten. Ich verstehe dieses Vorgehen als eine wissenschaftsnahe Variante künstlerischer Forschung, die im Rahmen von transformativen Methoden wie Realexperimenten und Reallaboren operiert.

\footnotetext{
7 Dieses vor allem für die moderne Wissenschaft charakteristische Ideal ist in der Praxis ohnehin nicht einzulösen, da Wissenschaft als menschliche Tätigkeit immer auch sozialen Bedingungen unterliegt und daher nicht vollkommen objektiv sein kann (Bellinger/ Krieger 2006: 18). Das einflussnehmende und normative Konzept von Realexperimenten und Reallaboren verbindet wissenschaftliche Forschung allerdings mit politischem Handeln im Sinne von zielgerichteten Eingriffen zur Beeinflussung einer nachhaltigen Transformation der Gesellschaft (Strohschneider 2014: 186). Dies trifft den Kern einer aufkommenden transformativen Wissenschaft. Diese noch nicht klar definierte und aufgrund ihrer normativen Ausrichtung kritisierte Disziplin (Strohschneider 2014: 186) soll aktiv und unmittelbar zur „Zukunftsgestaltung“ (Opielka 2017: 25) beitragen und muss dafür in gewissem Maße auch parteiisch sein in Bezug auf gesellschaftliche Veränderungsprozesse (Schneidewind 2015: 19).
} 


\section{Fazit}

Wie dieser Beitrag nahelegt, können Forschende Transformationswissen generieren, das auch Wertvorstellungen und Handlungsrationalitäten der Beteiligten umfasst, indem sie künstlerische Praktiken im Rahmen von Realexperimenten einsetzen. Vor allem aber können auf diese Weise bei den Beteiligten Erfahrungen kreiert werden, die gemeinsame Lernprozesse anstoßen und helfen, Empathie und Vertrauen unter ihnen aufzubauen. Dadurch können sie motiviert und in die Lage versetzt werden, aktiv im Sinne einer nachhaltigen Transformation zu handeln. Diese Wirkungen lassen sich durch die Forschenden allerdings nicht sicherstellen. Ihre Interventionen können auch wirkungslos bleiben oder sogar unbeabsichtigte Folgen haben, die einer nachhaltigen Transformation zuwiderlaufen. Auch lässt sich durch die Einbindung von künstlerischen Praktiken nicht das methodische Spannungsverhältnis von Reallaboren auflösen, dass einerseits über Realexperimente überraschendes Wissen (ko)produziert werden soll und sie dementsprechend ergebnisoffen zu gestalten sind, und dass andererseits über Reallabore Transformationsprozesse zielgerichtet und entsprechend der normativen Grundannahmen der Forschenden zu steuern sind. Ebenso bleiben andere für Reallabore typische Herausforderungen wie der Umgang mit Doppelrollen, das transdisziplinäre Gestalten von Forschungsdesigns ( $\mathrm{Co}-\mathrm{De}$ sign) und der produktive Umgang mit Nichtwissen bestehen. Solche Herausforderungen können sogar noch vergrößert werden, wenn der Einsatz von künstlerischen Praktiken für die Beteiligten ungewohnt ist und sie sich deshalb nicht darauf einlassen möchten, etwa weil sie von deren Potenzialen erst noch überzeugt werden müssen oder weil sie mit ihrer Rolle als Teilnehmende einer Inszenierung oder Performance hadern, bei der sie aktiv einbezogen werden, die sie aber nicht kontrollieren können.

Der produktive Umgang mit solchen Herausforderungen erfordert von den Forschenden neben ausreichenden Zeiträumen vor allem eine hohe soziale Kompetenz, denn sie müssen die Beteiligten dauerhaft einbinden, den Forschungsprozess gegebenenfalls an deren Belange und Handlungszwänge anpassen und Zwischenergebnisse so in soziale Prozesse einspeisen, dass diese Anwendung finden. Die Fähigkeit zum zielgerichteten Einsatz von künstlerischen Praktiken bei der Gestaltung und Durchführung von Realexperimenten schließt insofern auch ebendiese soziale Kompetenz mit ein, wie auch den Willen der Forschenden, selbst aktiv an der Gestaltung von Transformationsprozessen mitzuwirken.

Danksagung Der Beitrag basiert auf der Promotionsschrift „Koproduktion Urbaner Resilienz - Das Gängeviertel in Hamburg als Reallabor für eine zukunftsfähige Stadtentwicklung von Zivilgesellschaft, Politik und Verwaltung“. Diese geht zurück auf das Graduiertenkolleg Per- forming Citizenship, das von der Landesforschungsförderung Hamburg gefördert und von folgenden Institutionen getragen wurde: Forschungstheater / Fundus Theater, K3 - Zentrum für Choreographie | Tanzplan Hamburg, Hochschule für Angewandte Wissenschaften Hamburg, HafenCity Universität Hamburg.

\section{Literatur}

Badura, J.; Dubach, S.; Haarmann, A. (2015): Warum ein Handbuch zur künstlerischen Forschung? In: Badura, J.; Dubach, S.; Haarmann, A.; Mersch, D.; Rey, A.; Schenker, C.; Toro Pérez, G. (Hrsg.): Künstlerische Forschung. Ein Handbuch. Zürich, 9-16.

Beecroft, R.; Trenks, H.; Rhodius, R.; Benighaus, C.; Parodi, O. (2018): Reallabore als Rahmen transformativer und transdisziplinärer Forschung. Ziele und Designprinzipien. In: Defila, R.; Di Guilio, A. (Hrsg.): Transdisziplinär und transformativ forschen. Eine Methodensammlung. Wiesbaden, 75-100. https://doi.org/10.1007/9783-658-21530-9_4

Belina, B. (2013): Raum. Zu den Grundlagen eines historisch-geographischen Materialismus. Münster.

Belliger, A.; Krieger, D. J. (2006): Einführung in die Akteur-Netzwerk-Theorie. In: Belliger, A.; Krieger, D. J. (Hrsg.): ANThology. Ein einführendes Handbuch zur Akteur-Netzwerk-Theorie. Bielefeld, 13-50.

Berger, H. (2018): Handlung statt Verhandlung. Kunst als gemeinsame Stadtgestaltung. Berlin.

Berger, H. M.; Ziemer, G. (2017): New Stakeholders of Urban Change. A Question of Culture and Attitude? In: Berger, H. M.; Ziemer, G. (Hrsg.): New Stakeholders of Urban Change. A Question of Culture and Attitude? Berlin, 11-20. $=$ Perspectives in Metropolitan Research 4.

Bippus, E. (2009): Einleitung. In: Bippus, E. (Hrsg.): Kunst des Forschens. Praxis eines ästhetischen Denkens. Zürich, 7-23.

Böhme, G. (2011): Atmosphären. In: Hauser, S.; Kamleithner, C.; Meyer, R. (Hrsg.): Architekturwissen. Grundlagentexte aus den Kulturwissenschaften. Band 1: Zur Ästhetik des sozialen Raumes. Bielefeld, 236-246.

Bovaird, T.; Loeffler, E. (2016): Bringing the Resource of Citizens into Public Governance. Innovation through Co-Production to Improve public Services and Outcomes. In: Torfing, J.; Triantafillou, P. (Hrsg.): Enhancing Public Innovation by Transforming Public Governance. Cambridge, 160-177. https://doi.org/10.1017/ CBO9781316105337.008

Brand, U.; Wissen, M. (2017): Imperiale Lebensweise. Zur Ausbeutung von Mensch und Natur im globalen Kapitalismus. München. 
Breckner, I. (2015): Stadterneuerung in Eigenregie. Das sozio-kulturelle Realexperiment im Hamburger Gängeviertel. In: Bezirksamt Pankow von Berlin (Hrsg.): Eine Stadt verändert sich. Berlin Pankow. 25 Jahre Stadterneuerung. Berlin, 188-199.

Bruns, T. (2018): Linke Zentren - das Rückgrat der Proteste. In: GoGoGo (Hrsg.): Das war der Gipfel. Die Proteste gegen G20 in Hamburg. Berlin, 56-71.

BSU - Behörde für Stadtentwicklung und Umwelt der Freien und Hansestadt Hamburg (2010): Integriertes Entwicklungskonzept Gängeviertel/Valentinskamp. Hamburg.

Defila, R.; Di Guilio, A. (2018a): Partizipative Wissenserzeugung und Wissenschaftlichkeit - ein methodologischer Beitrag. In: Defila, R.; Di Guilio, A. (Hrsg.): Transdisziplinär und transformativ forschen. Eine Methodensammlung. Wiesbaden. 39-67. https://doi.org/10. 1007/978-3-658-21530-9_2

Defila, R.; Di Guilio, A. (2018b): Reallabore als Quelle für die Methodik transdisziplinären und transformativen Forschens - eine Einführung. In: Defila, R.; Di Guilio, A. (Hrsg.): Transdisziplinär und transformativ forschen. Eine Methodensammlung. Wiesbaden, 9-35. https://doi. org/10.1007/978-3-658-21530-9_1

Eckardt, F.; Brokow-Loga, A. (2020): Einleitung. Der sozial-ökologische Wandel der Stadtgesellschaft. In: Brokow-Loga, A.; Eckardt, F. (Hrsg.): Postwachstumsstadt. Konturen einer solidarischen Stadtpolitik. München, 1427. https://doi.org/10.14512/9783962386962

Engel, T.; Knieling, J. (2018): „Große Transformation“ und nachhaltige Raumentwicklung. Stand der Diskussion und theoretische Zugänge. In: Knieling, J. (Hrsg.): Wege zur großen Transformation. Herausforderungen für eine nachhaltige Stadt- und Regionalentwicklung. München, 13-32.

Folke, C. (2016): Resilience. In: Oxford Research Encyclopedia of Environmental Science. Oxford, 1-66. https:// doi.org/10.1093/acrefore/9780199389414.013.8

Frahm, O. (2015): Intervenieren. In: Badura, J.; Dubach, S.; Haarmann, A.; Mersch, D.; Rey, A.; Schenker, C.; Toro Pérez, G. (Hrsg.): Künstlerische Forschung. Ein Handbuch. Zürich,165-168.

Frantzeskaki, N.; Dumitru, A.; Anguelovski, I.; Avelino, F.; Bach, M.; Best, B.; Binder, C.; Barnes, J.; Carrus, G.; Egermann, M.; Haxeltine, A.; Moore, M.-L.; Garcia Mira, R.; Loorbach, D.; Uzzell, D.; Omann, I.; Olsson, P.; Silvestri, G.; Stedman, R.; Wittmayer, J.; Durrant, R.; Rauschmayer, F. (2016): Elucidating the Changing Roles of Civil Society in Urban Sustainability Transitions. In: Current Opinion in Environmental Sustainability 22, 41-50. https://doi.org/10.1016/j.cosust.2017.04.008

Frantzeskaki, N.; Haase, D.; Fragkias, M.; Elmqvist, T.
(2016): Editorial Overview. System Dynamics and Sustainability. Urban Transitions to Sustainability and Resilience. In: Current Opinion in Environmental Sustainability 22, iv-viii. https://doi.org/10.1016/j.cosust.2017. 05.001

Gilbert, A. (2016): Publishing as Artistic Practice. In: Gilbert, A. (Hrsg.): Publishing as Artistic Practice. Berlin, 6-39.

Groß, M. (2014): Experimentelles Nichtwissen. Umweltinnovationen und die Grenzen sozial-ökologischer Resilienz. Bielefeld. https://doi.org/10.14361/transcript. 9783839428559

Groß, M. (2017): Experimentelle Kultur und die Governance des Nichtwissens. In: Reinermann, J.-L.; Behr, F. (Hrsg.): Die Experimentalstadt. Kreativität und die kulturelle Dimension der Nachhaltigen Entwicklung. Wiesbaden, 21-40. https://doi.org/10.1007/978-3-65814981-9_2

Gualini, E. (2010): Zivilgesellschaftliches Handeln und bürgerschaftliches Engagement aus stadtentwicklungspolitischer Perspektive. Kritische Überlegungen zur Thematik. In: Becker, E.; Gualini, E.; Runkel, C.; Graf Strachwitz, R. (Hrsg.): Stadtentwicklung, Zivilgesellschaft und bürgerschaftliches Engagement. Stuttgart, 3-22. https://doi. org/10.1515/9783110507867-002

Haarmann, A. (2019): Artistic Research. Eine epistemologische Ästhetik. Bielefeld. https://doi.org/10.14361/ 9783839446362

Hahne, U. (2018): Die Region in der Postwachstumsdebatte. In: Knieling, J. (Hrsg.): Wege zur großen Transformation. Herausforderungen für eine nachhaltige Stadt- und Regionalentwicklung. München, 49-64.

Hassler, U.; Kohler, N. (2014): Resilience in the built Environment. In: Building Research and Information 42, 2, 119-129. https://doi.org/10.1080/09613218.2014. 873593

Heinrichs, H. (2018): Sustainability Science with Ozzy Osbourne, Julia Roberts and Ai Weiwei. The Potential of arts-based Research for Sustainable Development. In: GAIA - Ecological Perspectives for Science and Society 27, 1, 132-137. https://doi.org/10.14512/gaia.27.1.8

Hildebrandt, P. (2014): „The Researcher Is Present“. Künstlerische Formen der Wissensproduktion in den Sozialwissenschaften. In: Stemmler, S. (Hrsg.): Wahrnehmung, Erfahrung, Experiment, Wissen. Objektivität und Subjektivität in den Künsten und den Wissenschaften. Zürich, 71-80.

Holub, B. (2015): Planning Unplanned. Towards a new Positioning of Art in the Context of Urban Development. In: Holub, B.; Hohenbüchler, C. (Hrsg.): Planning Unplanned. Towards a New Function of Art in Society. Wien, $20-46$. 
Jackson, T. (2017): Wohlstand ohne Wachstum - Das Update. Grundlagen für eine zukunftsfähige Wirtschaft. München.

Kagan, S.; Kirchberg, V.; Weisenfeld, U. (2019): Stadt als Möglichkeitsraum - Möglichkeitsräume in der Stadt. Eine Einführung. In: Kagan, S.; Kirchberg, V.; Weisenfeld, U. (Hrsg.): Stadt als Möglichkeitsraum. Experimentierfelder einer urbanen Nachhaltigkeit. Bielefeld, 15-35. https://doi.org/10.14361/9783839445853-002

Kopatz, M. (2016): Ökoroutine. Damit wir tun, was wir für richtig halten. München.

Krohn, W. (2012): Künstlerische und wissenschaftliche Forschung in transdisziplinären Projekten. In: Tröndle, M.; Warmers, J. (Hrsg.): Kunstforschung als ästhetische Wissenschaft. Beiträge zur transdisziplinären Hybridisierung von Wissenschaft und Kunst. Bielefeld, 1-19. https://doi. org/10.14361/transcript.9783839416884.1

Lamker, C.; Schulze Dieckhoff, V. (2020a): Postwachstum + Planung = Postwachstumsplanung?! Erfahrungen aus der Konfrontation zweier Diskurse. In: Brokow-Loga, A.; Eckardt, F. (Hrsg.): Postwachstumsstadt. Konturen einer solidarischen Stadtpolitik. München, 90-103. https:// doi.org/10.14512/9783962386962

Lamker, C.; Schulze Dieckhoff, V. (2020b): Neue Rollen kollektiver wachstumsunabhängiger Raumorganisation. In: Lange, B.; Hülz, M.; Schmid, B.; Schulz, C. (Hrsg.): Postwachstumsgeographien. Raumbezüge diverser und alternativer Ökonomien. Bielefeld, 369-386. https://doi. org/10.14361/9783839451809-026

Lessenich, S. (2018): Neben uns die Sintflut. Wie wir auf Kosten anderer Leben. München.

Malorny, T. (2018): Wirklichkeitsperforationen. Wege angewandter künstlerischer Zukunftsforschung. In: Förster, M.; Hebert, S.; Hofmann, M.; Jonas, W. (Hrsg.): Un/ Certain Futures. Rollen des Designs in gesellschaftlichen Transformationsprozessen. Bielefeld, 74-84. https://doi. org/10.14361/9783839443323-008

Matthias, S.; Wildner, K. (2021): Intervention into the Real. https://pab-research.de/intervention-into-the-real/ (27.01.2021).

McPhearson, T.; Pickett, S.; Grimm, N. B.; Niemelä, J.; Alberti, M.; Elmqvist, T.; Weber, C.; Haase, D.; Breuste, J.; Qureshi, S. (2016): Advancing Urban Ecology toward a Science of Cities. In: Bio Science 66, 3, 198-212. https://doi.org/10.1093/biosci/biw002

Meerow, S.; Newell, J. P.; Stults, M. (2016): Defining Urban Resilience. A Review. In: Landscape and Urban Planning 147, 38-49. https://doi.org/10.1016/j.landurbplan.2015. 11.011

Novy, J.; Colomb, C. (2013): Struggling for the Right to the (creative) City in Berlin and Hamburg: New Urban Social Movements, New 'Spaces of Hope'? In: Internatio- nal Journal of Urban and Regional Research 37, 5, 18161838. https://doi.org/10.1111/j.1468-2427.2012.01115.x

Olsson, P.; Galaz, V.; Boonstra, W. (2014): Sustainability Transformations. A Resilience Perspective. In: Ecology and Society 19, 4, 1. https://doi.org/10.5751/ES-06799190401

Opielka, M. (2017): Soziale Nachhaltigkeit. Auf dem Weg zur Internalisierungsgesellschaft. München.

Ostrom, E. (1996): Crossing the Great Divide. Coproduction, Synergy and Development. In: World Development 24, 6, 1073-1087. https://doi.org/10.1016/0305750X(96)00023-X

Page, G. G.; Wise, R. M.; Lindenfeld, L.; Moug, P.; Hodgson, A.; Wyborn, C.; Fazey, I. (2016): Co-designing Transformation Research. Lessons learned from Research on deliberate Practices for Transformation. In: Current Opinion in Environmental Sustainability 20, 8692. https://doi.org/10.1016/j.cosust.2016.09.001

Peters, S. (2011): Der Vortrag als Performance. Bielefeld. https://doi.org/10.14361/transcript.9783839417744

Sanierungsbeirat Gängeviertel (2016): Sanierungsgebiet Gängeviertel/Valentinskamp. Protokoll der 10. Sitzung des Sanierungsbeirates am 22. Februar 2016. Hamburg.

Schäfer, M. (2013): Inter- und transdisziplinäre Nachhaltigkeitsforschung. Innovation durch Integration? In: Rückert-John, J. (Hrsg.): Soziale Innovation und Nachhaltigkeit: Perspektiven sozialen Wandels. Wiesbaden, 171-194. https://doi.org/10.1007/978-3-531-18974-1_ 10

Schäpke, N.; Stelzer, F.; Bergmann, M.; Singer-Brodowski, M.; Wanner, M.; Caniglia, G.; Lang, D. J. (2017): Reallabore im Kontext transformativer Forschung. Ansatzpunkte zur Konzeption und Einbettung in den internationalen Forschungsstand. Lüneburg. = IETSR Discussion Papers in Transdisciplinary Sustainability Research 1/2017.

Schmid, B. (2020): Räumliche Strategien für eine Postwachstumstransformation. In: Lange, B.; Hülz, M.; Schmid, B.; Schulz, C. (Hrsg.): Postwachstumsgeographien. Raumbezüge diverser und alternativer Ökonomien. Bielefeld, 59-83. https://doi.org/10.14361/ 9783839451809-005

Schneidewind, U. (2015): Verantwortung für die Gesellschaft. Wissenschaft in der Großen Transformation. In: Politische Ökologie 1, 18-23.

Schneidewind, U. (2018): Die Große Transformation. Eine Einführung in die Kunst gesellschaftlichen Wandels. Frankfurt am Main.

Schneidewind, U.; Scheck, H. (2013): Die Stadt als „Reallabor" für Systeminnovation. In: Rückert-John, J. (Hrsg.): Soziale Innovation und Nachhaltigkeit: Perspektiven sozialen Wandels. Wiesbaden, 230-248. https://doi.org/10. 1007/978-3-531-18974-1_10 
Schneidewind, U.; Singer-Brodowski, M. (2014): Transformative Wissenschaft: Klimawandel im deutschen Wissenschafts- und Hochschulsystem. Marburg.

Schulz, C.; Lange, B.; Hülz, M.; Schmid, B. (2020): Postwachstumsgeographien. Konzeptionelle und thematische Eckpunkte der Anthologie. In: Schulz, C.; Lange, B.; Hülz, M.; Schmid, B. (Hrsg.): Postwachstumsgeographien. Raumbezüge diverser und alternativer Ökonomien. Bielefeld, 13-32. https://doi.org/10.14361/ 9783839451809-003

Seel, M. (2011): Inszenieren als Erscheinenlassen. In: Hauser, S.; Kamleithner, C.; Meyer, R. (Hrsg.): Architekturwissen. Grundlagentexte aus den Kulturwissenschaften. Band 1: Zur Ästhetik des sozialen Raumes. Bielefeld, 352-358.

Selle, K. (2005): Planen. Steuern. Entwickeln. Über den Beitrag öffentlicher Akteure zur Entwicklung von Stadt und Land. Dortmund.

Selle, K. (2010): Die Koproduktion des Stadtraumes. Neue Blicke auf Plätze, Parks und Promenaden. In: Forum Wohnen und Stadtentwicklung 2, 59-65.

Sharifi, A.; Chelleri, L.; Fox-Lent, C.; Grafakos, S.; Pathak, M.; Olazabal, M.; Moloney, S.; Yumagulova, L.; Yamagata, Y. (2017): Conceptualizing Dimensions and Characteristics of Urban Resilience. Insights from a Co-Design Process. In: Sustainability 9, 1-20. https://doi.org/ $10.3390 / \mathrm{su} 9061032$

Siegmund, J. (2015): Formieren/Arrangieren. In: Badura, J.; Dubach, S.; Haarmann, A.; Mersch, D.; Rey, A.;
Schenker, C.; Toro Pérez, G. (Hrsg.): Künstlerische Forschung. Ein Handbuch. Zürich, 139-142.

Strohschneider, P. (2014): Zur Politik der Transformativen Wissenschaft. In: Brodocz, A.; Herrmann, D.; Schmidt, R.; Schulz, D.; Schulze Wessel, J. (Hrsg.): Die Verfassung des Politischen. Festschrift für Hans Vorländer. Wiesbaden, 175-192. https://doi.org/10.1007/9783-658-04784-9_10

Wanner, M.; Hilger, A.; Westerkowski, J.; Rose, M.; Stelzer, F.; Schäpke, N. (2018): Towards a Cyclical Concept of Real-World Laboratories. A Transdisciplinary Research Practice for Sustainability Transitions. In: disP - The Planning Review 54, 2, 94-114. https://doi.org/10.1080/ 02513625.2018 .1487651

Werner, S. (2012): Steuerung von Kooperationen in der integrierten und sozialen Stadtentwicklung. Machtverhältnisse und Beteiligung im Prozessraum. Wiesbaden. https:// doi.org/10.1007/978-3-531-19737-1

Ziehl, M. (2017): Zukunftsfähigkeit durch Kooperation. Ein Laborbericht aus dem Gängeviertel. Hamburg. http:// urban-upcycling.de/laborbericht (26.02.2021).

Ziehl, M. (2020): Koproduktion Urbaner Resilienz. Das Gängeviertel in Hamburg als Reallabor für eine zukunftsfähige Stadtentwicklung von Zivilgesellschaft, Politik und Verwaltung. Berlin.

Ziemer, G. (2019): Ein weißes Pferd. Kunst im Reallabor Stadt. In: Kunstforum International 262, 96-103. 